\title{
Cirugía endoscópica nasosinusal y de base de cráneo asistida por navegación
}

\author{
Endoscopic sinonasal surgery and skull base surgery assited navigation \\ Rodolfo Nazar S $^{1}$, Lucas Ritacco², Romina Gianini $\mathbf{V}^{1}$, Natalia Cabrera $\mathbf{S}^{1}$, \\ Carolina Oliva G', Alfredo Naser G'.
}

\begin{abstract}
RESUMEN
Introducción: La cirugía guiada por imágenes corresponde a una herramienta quirúrgica que comienza a desarrollarse a mediados del siglo XX. En 1990 comienza a utilizarse en otorrinolaringología, siendo sus principales indicaciones la cirugía endoscópica de cavidades paranasales y la cirugía de base de cráneo anterior.

objetivo: Analizar y presentar el resultado del uso de cirugía guiada por imágenes en pacientes intervenidos en el Hospital Clínico de la Universidad de Chile (HCUCH).

Material y método: Se realizó un estudio retrospectivo incluyendo todos los casos de cirugía endoscópica nasosinusal y base de cráneo asistida por imágenes en HCUCH. La selección para usar la navegación se hizo en base a las recomendaciones de la Academia Americana de Otorrinolaringología.

Resultados: De un total de 12 pacientes intervenidos con el uso de navegación, se obtuvo $100 \%$ de éxito quirúrgico con disminución de los síntomas y sin complicaciones intraoperatorias.

Conclusión: La cirugía asistida por navegación en cavidades paranasales y base de cráneo, es una herramienta de suma utilidad en casos seleccionados que potencia las habilidades quirúrgicas del cirujano sin reemplazar sus conocimientos anatómicos. Otorga seguridad y precisión en cirugías que comprometen estructuras nobles como base de cráneo y órbita entre otros.
\end{abstract}

Palabras clave: Cirugía asistida por imágenes, navegación, cirugía.

\begin{abstract}
Introduction: Image Guided Surgery is a surgical tool that develop in the mid-twentieth century. In 1990 it began to be used in otolaryngology, being its main indications endoscopic surgery of paranasal sinuses and anterior skull base.

Aim: To analyze and present the result of image-guided surgery in patients undergoing surgery at the Hospital of the University of Chile.
\end{abstract}

\footnotetext{
Médico Servicio Otorrinolaringología Hospital Clínico de la Universidad de Chile.

Médico Hospital Italiano de Buenos Aires.

3 Interna de Medicina, Facultad de Medicina Universidad de Chile.
} 
Material and method: A retrospective study included all cases of endoscopic sinus surgery and skull base imaging assisted at the Clinical Hospital of the University of Chile from August 2015 to August 2016. The choice to use the navigation was made in based on the recommendations of the American Academy of Otolaryngology, and analyzed case by case.

Results: A total of 12 patients were operated using Navigation, achieving $100 \%$ of surgical success with decreased symptoms and without intraoperative complications.

Conclusion: Image Guided Surgery in paranasal sinuses and skull base is a tool very useful in selected cases where enhances the surgeon skills without replacing their anatomical knowledge. Provides security and accuracy in surgeries involving noble structures such as the skull base, orbit among others. their anatomical knowledge. Provides security and accuracy in surgeries involving noble structures such as the skull base, orbit among others.

Key words: Image guided surgery, Navigation, Endoscopic sinonasal.

\section{INTRODUCCIÓN}

La cirugía guiada por imágenes corresponde a una herramienta quirúrgica que nace en el área de la neurocirugía con la finalidad de orientar anatómicamente al cirujano en el abordaje de tumores cerebrales ${ }^{1}$. Consta de una torre

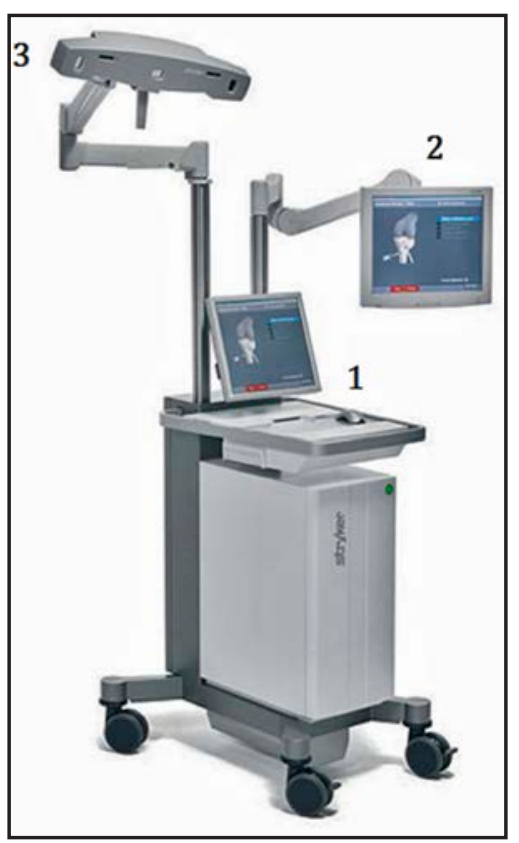

Figura 1. Navegador con sistema de comunicación óptico. Se observan sus diferentes componentes: 1. Estación computacional. 2. Monitor para el cirujano. 3. Sistema de comunicación óptico. computacional con un software especializado al que se cargan imágenes preoperatorias del paciente, las cuales son pareadas con puntos anatómicos de referencia durante el intraoperatorio y permite ubicarse con precisión durante la cirugía ${ }^{2}$ (Figura 1).

La primera aproximación se remonta al año 1947, cuando Spiegel y Wycis crean un sistema de cirugía estereotáxica con rayos $X$. Desde ese momento hasta la actualidad se ha incursionado en diversas técnicas de navegación, llegando incluso a softwares que permiten incorporar múltiples imágenes preoperatorias como tomografías, resonancias 0 angiografías otorgando mayor precisión en casos seleccionados. Es lo que se conoce como navegación multimodal. En 1990 se incorpora a algunos servicios de otorrinolaringología para ser un apoyo en cirugías de cavidades paranasales y base de cráneo con muy buenos resultados. En USA es utilizado por el $73 \%$ de los otorrinolaringólogos y $80 \%$ de éstos afirma que otorga mayor seguridad durante pasos críticos de la cirugía ${ }^{3}$.

\section{Navegador}

Como mencionamos anteriormente, el navegador se compone de diversos elementos (Figura 1). Presenta una estación computacional con un software especializado. En esta estación se cargan las imágenes preoperatorias del paciente que posteriormente serán emitidas por el monitor de la estación en sus 3 cortes: coronal, sagital y axial. Es 
requisito contar con una tomografía de cavidades paranasales de corte fino de $1 \mathrm{~mm}$ de todo el cráneo, con un protocolo de navegación del cual disponen los servicios de radiología actualmente.

Una vez en pabellón, se realiza el registro del paciente que corresponde a la conexión entre las imágenes preoperatorias y estructuras anatómicas claves o hitos de referencia anatómica. Luego de este proceso el navegador es capaz de traducir cualquier punto de la anatomía del paciente en un punto dentro de las imágenes.

Todos los navegadores requieren un registro en orden para poder emplear las imágenes preoperatorias. Los mejores puntos anatómicos para realizar este procedimiento son reparos óseos debido a su mayor precisión y menor tasa de errores. Existen 3 tipos de registro: el registro de puntos pareados, registro automático y registro basado en contornos. Los 3 han demostrado ser confiables con una precisión de 1-2 mm.

El sistema utilizado en nuestro servicio correspondió al sistema de puntos pareados, por medio de una máscara de superficie, que es lo que se observa en la Figura 2. Luego del registro viene el rastreo intraoperatorio, que se puede llevar a cabo mediante sistemas ópticos o electromagnéticos. En nuestro centro utilizamos la conexión mediante sistema óptico, que utiliza luz infrarroja y una cámara de rastreo posicionada sobre la cabeza del paciente que reconoce instrumental con esferas reflectivas. Esto permite localizar el puntero 0 el material quirúrgico especializado para la navegación y traducirlo a un punto en las imágenes de la tomografía. No existen diferencias significativas entre ambos métodos, obteniendo una precisión equivalente. La estación computacional integra la información recibida mediante los rastreos y proyecta la ubicación del puntero de navegación en los tres planos de la imagen preoperatoria.

Es importante que durante la cirugía el cirujano realice chequeos periódicos de la precisión del navegador, posicionando el puntero 0 material quirúrgico sobre puntos conocidos de la anatomía, frente a cualquier incongruencia observada entre la anatomía y lo que evidencia la tomografía. En nuestra experiencia esto no fue necesario, realizando solo la calibración inicial.

La navegación permite una planificación preoperatoria de la cirugía y es un apoyo imagenológico constante durante ésta. En ningún caso reemplaza los conocimientos anatómicos del cirujano, por el contrario, los complementa y los potencia. El rinólogo, debe tener una formación sólida de carácter teórico-práctico, con cursos de

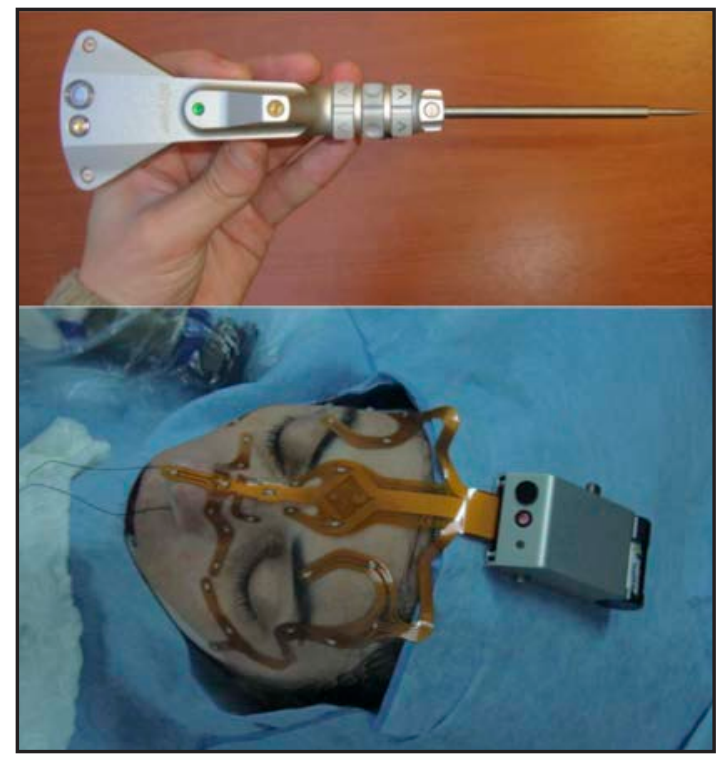

Figura 2. En la imagen superior se observa el puntero utilizado para realizar el rastreo intraoperatorio. En la imagen inferior se observa la máscara de silicona que permite realizar el registro de los puntos anatómicos. 
disección periódicos, que nunca serán reemplazados por la navegación.

\section{Indicaciones}

A pesar que las complicaciones de la cirugía endoscópica funcional de cavidades paranasales y base de cráneo ocurren en un bajo porcentaje de las intervenciones realizadas, generalmente corresponden a complicaciones graves, incluso fatales que generan la gran mayoría de los litigios en rinosinusología ${ }^{4}$. Es por esto, que la Academia Americana de Otorrinolaringología y Cirugía de Cabeza y Cuello recomienda el uso de navegador en ciertas intervenciones (Tabla 1).

Es necesario recordar que el uso de cirugía guiada por imágenes no mejora el resultado quirúrgico, solo ofrece mayor precisión y seguridad al cirujano sin reemplazar los conocimientos y entrenamiento quirúrgico previo, como ya hemos mencionado previamente ${ }^{5}$.

En nuestro centro se desarrollan alrededor de 150 cirugías endoscópicas nasosinusales al $a \tilde{n} 0^{6}$, en nuestra casuística se presentan 12 casos de Cirugía Endoscópica Nasosinusal asistida por navegación, lo que determina $8 \%$ de selección para el uso de esta herramienta debido a casos de anatomía compleja o alterada, resección de tumores nasosinusales y estrecha relación con estructuras nobles como órbita y base de cráneo.

\section{OBJETIVO}

Presentar y analizar el resultado del uso de cirugía guiada por imágenes en pacientes intervenidos en el Hospital Clínico de la Universidad de Chile.

\section{MATERIAL Y METODO}

Se presenta un estudio descriptivo retrospectivo llevado a cabo en el Servicio de Otorrinolaringología del Hospital Clínico de la Universidad de Chile, desde agosto del año 2015 hasta agosto del 2016. Fueron incluidos en este estudio todos los pacientes en los cuales fue utilizado el navegador, con un total de 12 cirugías de cavidades paranasales y de base de cráneo asistida por imágenes. La indicación quirúrgica y la utilidad del navegador en cada procedimiento se resumen en la Tabla 2. De los 12 pacientes, 9 fueron del sexo masculino ( $75 \%$ ) y 3 pacientes de sexo femenino $(25 \%)$, con un rango de edad desde los 13 a 69 años, con una media de 47 años. Las patologías por las cuales los pacientes se sometieron al procedimiento quirúrgico fueron: 2 fístulas de LCR (16.7\%), 1 osteoma etmoidoorbitario (8,3\%), 1 cuerpo extraño nasosinusal odontógeno (8,3\%), 1 seno silente con enoftalmo $(8,3 \%)$, 1 papiloma invertido operado $(8,3 \%), 2$ rinosinusitis crónicas operadas $(16,7 \%)$, 2 rinosinusitis crónicas frontal (RSC) $(16,7 \%)$ y 2 rinosinusitis crónica poliposas $(16,7 \%)$.

Tabla 1. Indicaciones de cirugía guiada por imágenes según la AAO-HNS ${ }^{6}$

\section{Indicaciones de Cirugía Guiada pHNS}

Cirugía de revisión de cavidades paranasales

Anatomía alterada de cavidades paranasales (alteración del desarrollo, posoperatoria o de origen traumático

Poliposis nasosinusal masiva

Patología que comprometa seno frontal, etmoides posterior y seno esfenoidal

Enfermedad que colinde con la base de cráneo, órbita, nervio óptico y arteria carótida

Rinulicuorrea (por fístula de líquido cefalorraquídeo) o condiciones en que exista un defecto de la base de cráneo

Neoplasia de origen rinosinusal benigna o maligna 
Tabla 2. Resumen de los 12 casos intervenidos en nuestro hospital con ayuda de navegador. Se ordenan de forma cronológica, según diagnóstico y utilidad del navegador

\begin{tabular}{|c|c|c|}
\hline Casos & Diagnóstico & Utilidad de navegación \\
\hline 1 & Fístula de LCR lámina cribosa & $\begin{array}{l}\text { Localización defecto } \\
\text { Delimitar la extensión óseo defecto } \\
\text { Futuro: evitar fluorosceína }\end{array}$ \\
\hline 2 & Fístula de LCR lámina cribosa & $\begin{array}{l}\text { Localización defecto } \\
\text { Delimitar la extensión óseo defecto } \\
\text { Futuro: evitar fluorosceína }\end{array}$ \\
\hline 3 & Rinosinusitis frontal crónica & $\begin{array}{l}\text { Límites óseos para fresar } \\
\text { Localización de celdilla interfrontal } \\
\text { Precisar sitio de frontotomía } \\
\text { Delimitar base de cráneo }\end{array}$ \\
\hline 4 & Pansinusitis crónica operada & $\begin{array}{l}\text { Confirmar reparos anatómicos alterados en CEF de revisión } \\
\text { Localización de seno frontal con diámetro AP pequeño } \\
\text { Localización de celdilla esfenoetmoidal }\end{array}$ \\
\hline 5 & Cuerpo extraño nasosinusal & $\begin{array}{l}\text { Localización precisa de cuerpo extraño odontogénico } \\
\text { Precisión y seguridad en inflamación severa }\end{array}$ \\
\hline 6 & Seno silente, enoftalmo & $\begin{array}{l}\text { Abordaje seguro del maxilar } \\
\text { Evitar penetrar a órbita por descenso de ella }\end{array}$ \\
\hline 7 & Papiloma invertido frontoetmoidal operado & $\begin{array}{l}\text { Límites óseos para fresar en sinusotomía frontal Draf III } \\
\text { Corroborar dehiscencia en órbita } \\
\text { Anatomía alterada en cirugía de revisión }\end{array}$ \\
\hline 8 & Pansinusitis crónica poliposa operada & $\begin{array}{l}\text { Confirmar reparos anatómicos alterados en CEF de revisión } \\
\text { Mayor seguridad en ubicar frontal } \\
\text { Hemostasia dificultosa durante la cirugía }\end{array}$ \\
\hline 9 & RSC poliposa pansinusal & $\begin{array}{l}\text { Precisión y seguridad en inflamación severa } \\
\text { Abordaje seguro del frontal y de esfenoides }\end{array}$ \\
\hline 10 & RSC frontal & $\begin{array}{l}\text { Localización precisa del receso del seno frontal } \\
\text { Seguridad para completar la frontotomía }\end{array}$ \\
\hline 11 & Osteoma etmoidoorbitario & $\begin{array}{l}\text { Resección completa del osteoma } \\
\text { Delimitación de base de cráneo y órbita } \\
\text { Precisar profundidad del fresado }\end{array}$ \\
\hline 12 & RSC poliposa & Confirmar reparos anatómicos alterados \\
\hline
\end{tabular}

\section{RESULTADOS}

De un total de 12 cirugías realizadas con ayuda del navegador, se obtuvo $100 \%$ de éxito quirúrgico con mejoría sintomática. Durante los procedimientos no se dañaron estructuras nobles y se pudieron identificar las estructuras anatómicas en los pasos críticos de las cirugías. El tiempo aproximado en armar la torre de navegación fue de 15 minutos, lo que no influyó en retrasos del tiempo quirúrgico. En las Figuras 3 a 6 se presentan imágenes de 4 casos clínicos y el uso de navegación.

\section{DISCUSIÓN}

La cirugía guiada por imágenes es utilizada a menudo para facilitar el entendimiento anatómico durante la cirugía, permitiendo discriminar detalles anatómicos finos en múltiples planos en tiempo real. Se ha postulado que por estas razones podría disminuir la frecuencia de complicaciones, sin embargo, la literatura no ha demostrado esta asociación ${ }^{8}$. Es importante considerar que la incidencia de complicaciones relacionadas con la CEF es muy baja, por lo que demostrar diferencias 


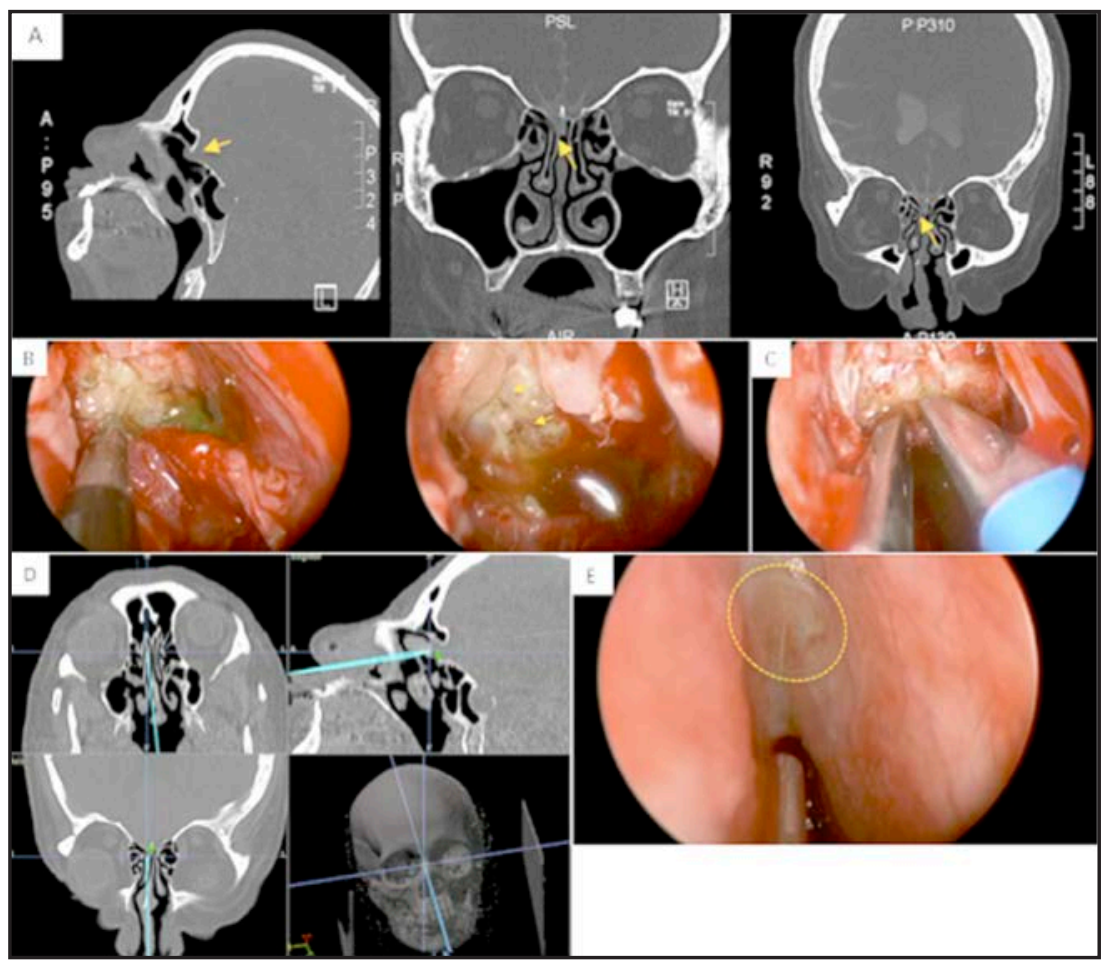

Figura 3. Fístula LCR, base de cráneo anterior derecha a nivel de la fóvea etmoidal asociado a meningocele. A. Tomografía computada y radiocisterno TAC con corte sagital y coronal que evidencia dehiscencia de lámina cribosa derecha, a nivel de la fóvea etmoidal y paso de medio de contraste desde la rinobase a la nariz. B. Imágenes de la visión endoscópica de la fístula. En la figura izquierda se observa escape de fluoresceína y en la figura derecha se observan las 2 brechas (flechas) en la duramadre luego de retirar la mucosa del techo etmoidal. C. Reducción del meningocele con bipolar. D. Captura de la pantalla del navegador que nos permite visualizar la imagen preoperatoria del paciente en su corte axial, coronal y sagital, más la visión endoscópica intraoperatoria. En este caso se observa el escape de fluoresceína en relación a la fistula de LCR de base de cráneo anterior, en la fóvea etmoidal. E. Se visualiza la dehiscencia a nivel del techo etmoidal solo cubierto por mucosa y que corresponde a la zona confirmada por navegación.

significativas con la navegación es un desafío, ya que el tamaño de la muestra de pacientes para mostrar un efecto significativo es excesivamente grande, necesitando enrolar 3.017 pacientes 9 .

En cuanto a sus ventajas la navegación permitiría realizar una planificación quirúrgica preoperatoria, ahorrar tiempo quirúrgico, una exploración más completa en un área potencialmente peligrosa manteniendo la indemnidad de estructuras nobles, favorecer la formación de futuros cirujanos y acortar su curva de aprendizaje, además de ser beneficiosa en la confirmación de la indemnidad quirúrgica de estructuras nobles en cirugía sinusal, especialmente en cirujanos menos experimenta$\operatorname{dos}^{10}$.

Se espera que el error objetivo razonable esté en el rango de 1,5 a 2,0 mm con esta herramienta, utilizándose navegación con realidad aumentada se ha estimado un error de $1,5 \mathrm{~mm} 0$ menos $^{11}$, en un estudio reciente el uso de navegación virtual 3D no demostró superioridad en comparación a una guía de imagen triplana convencional ${ }^{12}$. Se ha estimado que en nuestro centro el registro de error no supera $1 \mathrm{~mm}$.

Se ha comprobado que la cirugía guiada por imágenes en pacientes con variedad de patologías 


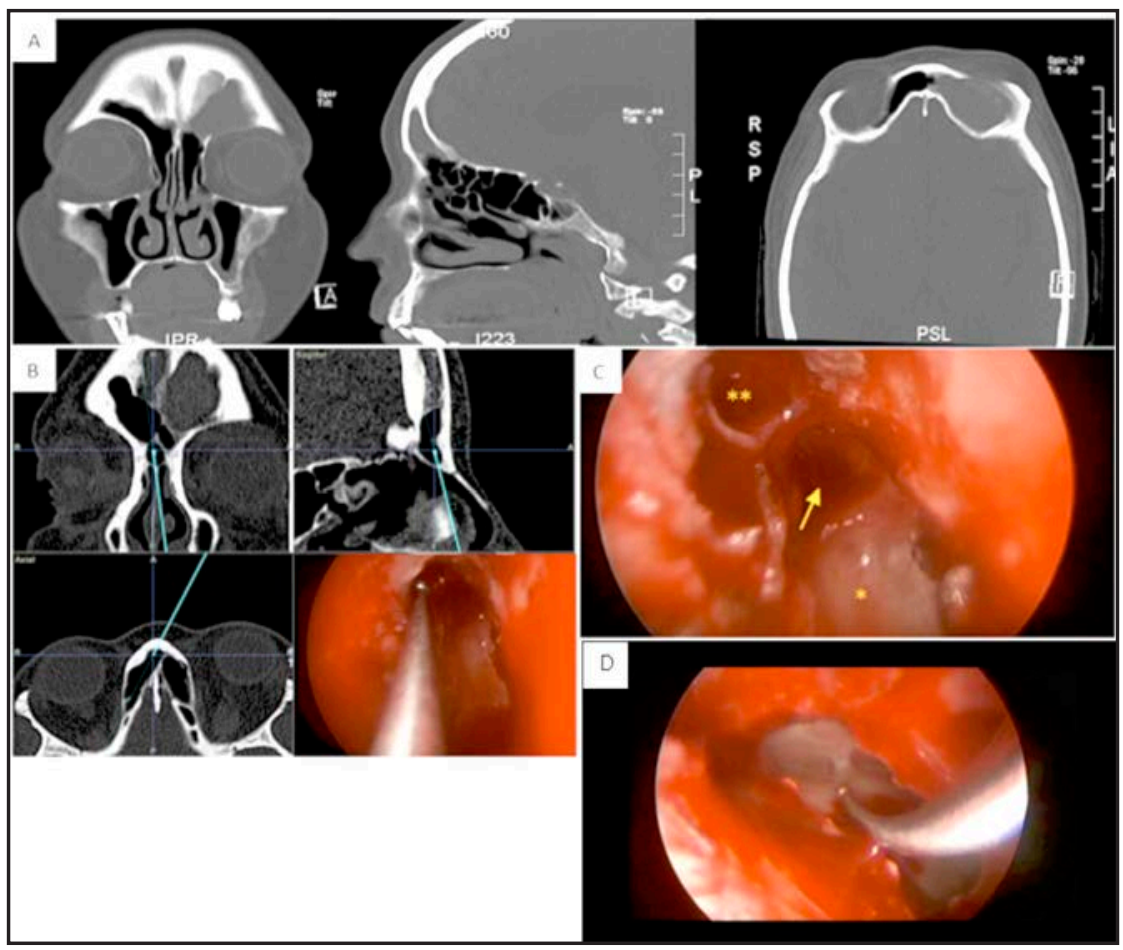

Figura 4. RSC con tabicación alta del seno frontal. Se realiza frontotomía endoscópica asistida por imágenes. A. Tomografía computada de cavidades paranasales en sus 3 cortes: Coronal, sagital y axial. Se evidencia ocupación de seno frontal a izquierda con tabique frontal alto. B y C. Visión endoscópica y visión de la navegación intraoperatoria. El identificar el receso del seno frontal corresponde a uno de los pasos críticos de la cirugía donde la navegación nos permite ingresar con seguridad al seno frontal. En la figura $\mathrm{C}$ la flecha nos indica el sitio de ingreso al receso del frontal, visualizándose hacia posterior la base de cráneo $\left({ }^{\star}\right)$, y hacia anterior una celdilla interseptal. $\left.{ }^{* *}\right)$. En la imagen B al navegar se observa la celdilla interseptal y el acceso al seno frontal por posterior a esta y por delante de la base de cráneo.D. Sinusostomía frontal tipo Draf IIB completada, se observa el puntero de navegación en la pared posterior del seno frontal.

nasosinusales con erosión de base de cráneo y/0 orbitaria sin extensión intracraneal 0 intraorbitaria es un procedimiento efectivo y seguro, permitiendo eliminar la morbilidad y mortalidad asociada a procedimientos abiertos y necesidad de reconstrucción ${ }^{13}$. En un estudio se comparó la esfenoidotomía en CEF de revisión con y sin navegación, encontrándose que la frecuencia de esfenoidotomía exitosa con cirugía guiada por imágenes fue sobre el $91 \%$ mientras que en la cirugía sin navegación, el porcentaje de éxito bajó a $68,7 \%$, demostrando una diferencia significativa ${ }^{14}$. En otra investigación cadavérica sobre abordaje lateral de base de cráneo, se midió el error de registro en 28 puntos de referencia quirúrgicos, analizando veinte huesos temporales de cadáveres mediante navegación electromagnética. Once de 17 objetivos (65\%) en el enfoque translaberíntico y cinco de 11 objetivos $(45 \%)$ en el enfoque de fosa media se identificaron, en el sistema de navegación, con error de registro de menos de $0,5 \mathrm{~mm}^{15}$. Concluyendo que el sistema de navegación electromagnético tiene una precisión suficiente para ser utilizado también en ciertos casos de cirugía de base de cráneo lateral.

Paradójicamente, un exceso de confianza podría incurrir en un error en el cirujano. En efecto, Stetler y cols ${ }^{16}$ llevó a cabo un estudio en el cual los alumnos parecían confiar en el sistema de navegación en más del $90 \%$ del tiempo y demostró un aumento significativo del deseo de asumir riesgos. 


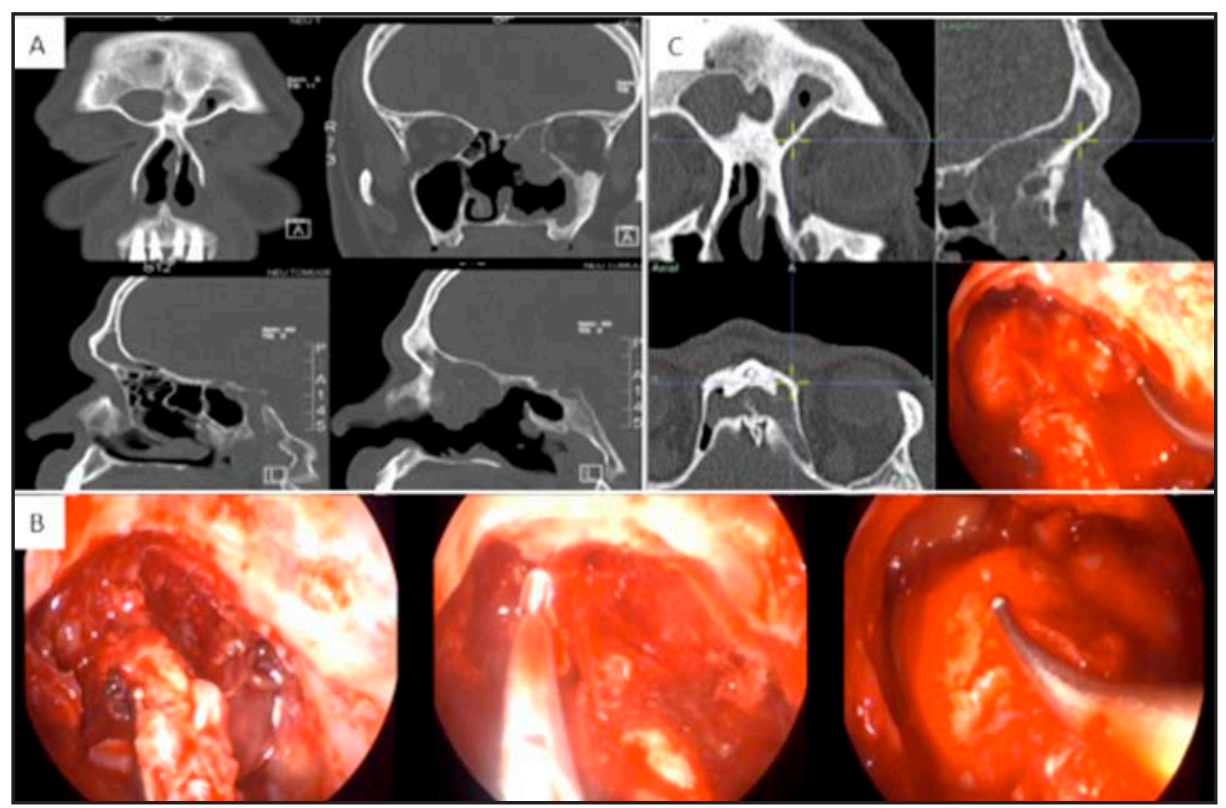

Figura 5. Papiloma invertido recidivado por segunda vez, con compromiso frontal y etmoidal. Se realiza sinusotomía frontal Draf III. A. Tomografía computada, se observa tumor nasosinusal izquierdo que presenta estrecha relación con órbita y base de cráneo. B. Visión endoscópica intraoperatoria de la sinusotomía tipo Draf III, en la imagen de la izquierda se observan ambos senos frontales y la septectomía posterior, en el centro se observa la resección del "beak del frontal" con pinza Cobra, a la derecha se muestra la clásica imagen en herradura del Draf III y el navegador sobre la base de cráneo. C. Navegación intraoperatoria que nos delimita el límite lateral de la resección, en el seno frontal izquierdo, donde había una dehiscencia hacia la órbita. Esto se sospechó durante la cirugía y se confirmó con la navegación.

En otro estudio el uso de una zona de proximidad de $3 \mathrm{~mm}$ (zonas de alerta) alrededor de las arterias carótidas y del nervio óptico, la distancia mínima fue de $1 \mathrm{~mm}$ sin navegación $(0,2$ a 3,1 $\mathrm{mm})$ y 0,6 $\mathrm{mm}$ con navegación $(0,2 \mathrm{a} 2,5 \mathrm{~mm})^{17}$. Por lo que el uso de sistemas guiados por imágenes intraoperatorias puede ser capaz de aumentar la confianza de manera subjetiva del cirujano en cuanto a la proximidad de estructuras críticas.

Encuestas a cirujanos han documentado mejoras en la carga de trabajo, el estrés, la orientación espacial y la confianza en la cirugía de base del cráneo, lo que implica lógicamente una mejora de los resultados. Para estudiar el estrés asociado a estos procedimientos se ha utilizado como parámetro la frecuencia cardiaca de cirujanos en formación. La carga mental durante la cirugía endoscópica funcional es alta en comparación a antes y después de la cirugía, y la navegación no demostró una diferencia significativa en el registro de la frecuencia cardiaca en comparación a la CEF sin navegación. Sin embargo, los residentes con experiencia y más de 30 procedimientos mostraron una ligera disminución en la carga mental utilizando navegación ${ }^{18}$.

Pese a todos los beneficios señalados, esta herramienta no reemplaza los conocimientos y la experiencia del cirujano y no está recomendada para la toma de decisiones intraoperatorias. Por lo que debe utilizarse sólo para confirmar o validar la sospecha anatómica o para reorientar cuando la visualización es subóptima o la anatomía no es sencilla, debiendo ser utilizada según criterio clínico y aplicado caso a caso.

Por otro lado, la navegación resulta de suma utilidad frente a hemostasia poco favorable, ya sea en casos de rinosinusitis crónica poliposa con inflamación severa o cirugía de tumores como papiloma invertido, en los cuales se deben hacer abordajes extendidos (Figura 5). En nuestra 


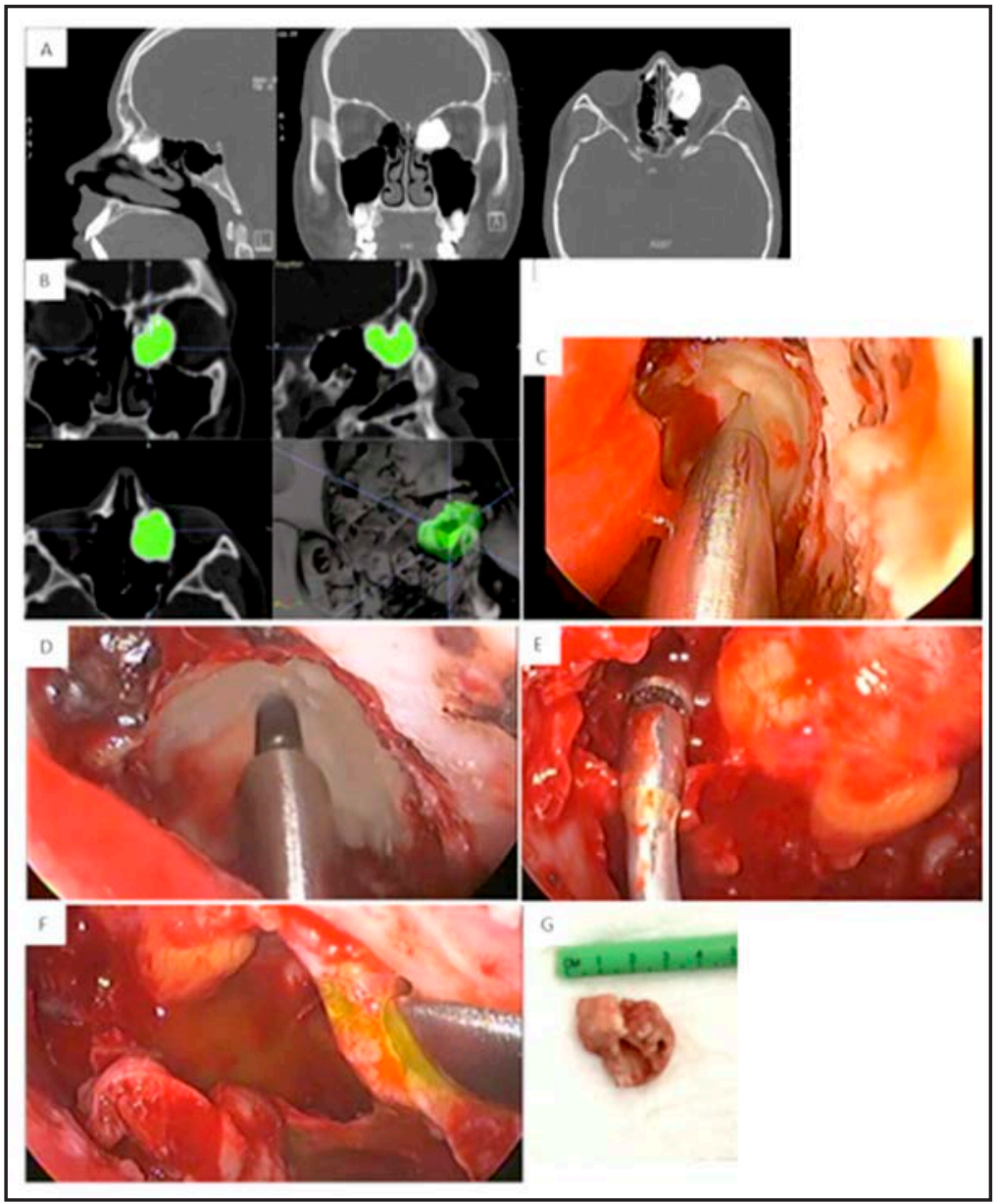

Figura 6. Osteoma frontoetmoidal. A. Tomografía computada de órbita y cavidades paranasales, plano sagital, coronal y axial. Se observa osteoma etmoidal izquierdo centrado en la lámina papirácea que mide aproximadamente $22 \times 30 \times 28 \mathrm{~mm}$ en sus diámetros transverso, anteroposterior y longitudinal, el que obstruye el receso naso-frontal izquierdo y el conducto naso-lagrimal, además de desplazar el músculo recto medial y el globo ocular, con moderada proptosis. En cortes sagital y coronal se observa estrecha relación del osteoma con la base de cráneo B. Navegación intraoperatoria con imágenes de planeamiento virtual preoperatorio. C. Se observa el puntero de la navegación en el espesor del osteoma para corroborar localización y profundidad del fresado. D. Fresado del osteoma con fresa de alta revolución (30.000 RPM). E Visión endoscópica de fosa nasal izquierda posresección del osteoma, se observa: grasa orbitaria que protruye a fosa nasal $\left({ }^{*}\right)$, receso del frontal permeable y ampliado $\left.{ }^{* *}\right)$. F. Visión endoscópica de fosa nasal izquierda posresección de osteoma, se observa: seno maxilar $\left({ }^{*}\right)$, conducto lagrimal permeable, al irrigar fluoresceína. $\left.{ }^{* \star}\right)$. G. Osteoma resecado en su totalidad luego del fresado.

casuística nos permitió corroborar la localización precisa de las 2 fístulas de líquido cefalorraquídeo (Figura 3), evaluar la profundidad del fresado en el caso del osteoma etmoidoorbitario (Figuras 6B6C) y delimitar los límites del fresado en el caso del papiloma invertido, en el que se realizó una sinusotomía frontal tipo Draf III (Figuras 5B-5C). En el osteoma además nos otorgó la funcionalidad del planeamiento virtual preoperatorio, lo cual permite una reconstrucción volumétrica del tumor y planificación de la resección en forma preoperatoria (Figura 6). En los casos de rinosinusitis crónica operada permitió corroborar la localización que sospechaba el cirujano al encontrarse la anatomía 
alterada. La cirugía asistida por navegación fue de extrema importancia en 2 casos de compromiso poliposo pansinusal, en la cual la hemostasia no fue la adecuada, a pesar de contar con TIVA y las medidas habituales de hemostasia, como cotonoides embebidos en epinefrina 1:20.000. Sin contar con esta herramienta la resección no hubiera sido la adecuada. En el caso de seno silente permitió un abordaje más seguro del seno maxilar al encontrarse la órbita descendida y en el caso del cuerpo extraño odontogénico nos permitió identificarlo con certeza, al encontrarse extruido en etmoides y al haber inflamación severa.

Por lo tanto, en nuestra experiencia las ventajas del uso de navegador fueron múltiples, otorgando al equipo mayor seguridad y precisión en el manejo de estos casos complejos, donde la cirugía es más extensa y demandante, debido al potencial

\section{BIBLIOGRAFÍA}

1. Palmer, James N., Chiu, Alexander G. Atlas of endoscopic sinus and skull base surgery. Elsevier Sauders (2013) 241-250.

2. WiSE S, DelGaudio J. Computer-aided surgery of the paranasal sinuses and skull base. Expert Rev. Med. Devices 2(4), 395-408 (2005).

3. RalPH M. Image-Guided Sinus Surgery: Lessons Learned from the first 1000 Cases. Otolaryngology Head and Neck Surgery 2003; 8-13.

4. Dalgorf D, Sacks R, Wormald PJ, Naidoo Y. Image-Guided Surgery influences perioperative morbidity from Endoscopic Sinus Surgery: A systematic review and meta-analysis. Otolaryngology-Head and Neck Surgery 149(1), 17-29 (2013).

5. Citardi M, Batra P. Intraoperative surgical navigation for endoscopic sinus surgery: rationale and indications. Current Opinion in Otolaryngology \& Head and Neck Surgery 2007, 15:23-2.

6. Cantillano P, Rubio F, Naser A, Nazar R. Cirugía Endoscópica Nasosinusal de Revisión. Acta Española de Otorrinolaringología Vol. 67. Núm 4. 2016. mayor riesgo de estructuras nobles, a diferencia de una cirugía endoscópica funcional habitual de cavidades paranasales.

\section{CONCLUSIONES}

El uso de cirugía guiada por imágenes disminuye el riesgo potencial ocasionado por factores inherentes a la orientación anatómica permitiendo discriminar detalles anatómicos en tiempo real, otorgando mayor seguridad en procedimientos complejos de cirugía endoscópica nasosinusal y de la rinobase.

Otorga una ayuda y un complemento al rinólogo en cirugía nasosinusal y de base de cráneo anterior, al permitir delimitar las zonas críticas, pero no reemplaza en ningún momento a la experiencia y conocimientos previos del cirujano.

7. AAO-HNS Policy on Intra-Operative use of Computer Aided Surgery (2005). American Academy of Otolaryngology-Head Neck Surgery. http://www. Entnet.Org/Practice/Policyintraoperativesurgery. $\mathrm{Cfm}$

8. Ramakrishnan VR, Kingdom TT. Does Image-Guided Surgery reduce complications? Otolaryngol Clin North Am 2015; 48(5): 851-9.

9. Smith TL, Stewart MG, Orlandi RR, Setzen M, LANZA DC. Indications for image-guided sinus surgery: the current evidence. Am J Rhinol 2007; 21: 80-3.

10. Strauss G, Koulechov K, Röttger S, Bahner J, Trantakis C, Hofer M, Korb W, Burgert 0, Meixensberger J, Manzey D, et al. Evaluation of a navigation system for ENT with surgical efficiency criteria. Laryngoscope 2006; 116: 56472.

11. Citardi MJ, Agbetoba A, Bigcas Jl, Luong A. Augmented reality for endoscopic sinus surgery with surgical navigation: a cadaver study. Int Forum Allergy Rhinol 2016; (5): 523-8. doi: 10.1002/alr.2170

12. Dixon BJ, Chan H, Daly MJ, Quu J, Vescan A, WITTERICK IJ, IRISH JC. Three-dimensional virtual 
navigation versus conventional image guidance: A randomized controlled trial. Laryngoscope 2016; 126(7): 1510-5. doi: 10.1002/lary.25882.

13. Al-QudaH M. Image-Guided Sinus Surgery in sinonasal pathologies with skull base/orbital erosion. J Craniofac Surg 2015; 26(5): 1606-8. doi: 10.1097/SCS.0000000000001841. Review.

14. JIANG RS, LIANG KL. Image-guided sphenoidotomy in revision functional endoscopic sinus surgery. Allergy Rhinol (Providence) 2014; 5(3): 116-9. doi: 10.2500/ar.2014.5.0093

15. Komune N, Matsushima K, Matsuo S, Safavi-Abbasi S, Matsumoto N, Rhoton AL JR. The accuracy of an electromagnetic navigation system in lateral skull base approaches. Laryngoscope 2016 Jun 15. doi: 10.1002/lary.25998.

16. Stelter K, Ertl-Wagner B, Luz M, Muller S, Ledderose G, Siedek V, Berghaus A, Arpe S, Leunig A.
Evaluation of an image-guided navigation system in the training of functional endoscopic sinus surgeons: a prospective, randomised clinical study. Rhinology 2011; 49: 429-37.

17. Haerle SK, Daly MJ, Chan H, Vescan A, Witterick I, Gentili F, Zadeh G, Kucharczyk W, IrISH JC. Localized intraoperative virtual endoscopy (LIVE) for surgical guidance in 16 skull base patients. Otolaryngol Head Neck Surg 2015; 152(1): 165-71. doi: 10.1177/0194599814557469.

18. Theodoraki MN, Ledderose GJ, Becker S, Leunig A, Arpe S, Luz M, Stelter K. Mental distress and effort to engage an image-guided navigation system in the surgical training of endoscopic sinus surgery: a prospective, randomised clinical trial. Eur Arch Otorhinolaryngol 2015; 272(4): 905-13. doi: 10.1007/s00405-0143194-0.

Dirección: Rodolfo Nazar S., Médico HCUCH

Santos Dumont \#999, Independencia Email : rnazars@gmail.com 